older patients in the community are not likely to be improved by 'term'-inally induced therapeutic nihilism. If we are to avoid such confusion, we should heed the words of another Lewis Carroll character:

" 'When I use a word,' Humpty Dumpty said in a
rather scornful tone, 'it means just what I choose
it to mean - neither more nor less.' "

Frank, E., Prien, R. F., Jarrett, R. B., et al (199I) Conceptualisation and rationale for consensus definitions of terms in major depressive disorder. Remission, recovery, relapse, and recurrence. Archives of General Psychiatry, 48, 85I-855.

Murphy, E. (1987) The prognosis of depression in old age (letter). British Journal of Psychiatry, 150, 268.

Tuma, T. A. (2000) Outcome of hospital-treated depression at 4.5 years. An elderly and a younger adult cohort compared. British Journal of Psychiatry, I76, 224-228.

D. Ridley-Siegert Cardiff and District NHS Trust, Royal Hamadryad Hospital, Cardiff Bay, Cardiff CFII 6UQ

\section{Anorexia nervosa: treatment with olanzapine}

Anorexia nervosa is a multiply determined disorder of unknown aetiology. Restriction of food intake culminating in profound emaciation is considered to be pathognomic (Kaye et al, 1999). One of the diagnostic criteria for anorexia nervosa is body image disturbance that is characterised by feeling and judging oneself to be fat and by claiming to 'see' oneself as fat despite being underweight. The bizarre body self-image in anorexia nervosa can be regarded as a psychotic way of thinking. We tried to treat anorexia nervosa as a psychotic disorder with olanzapine. Hansen (1999) has previously reported a case of treatment of anorexia nervosa with olanzapine; here we report three further cases.

A 50-year-old woman with anorexia nervosa since she was 17 years old applied for treatment in our clinic with an initial weight of $34 \mathrm{~kg}$ and height of $157 \mathrm{~cm}$. By that time she had already been treated with antidepressants, traditional neuroleptics and psychotherapy, without success. She was commenced on olanzapine, $5 \mathrm{mg}$ daily. The first 2 months were difficult because she had to accept the side-effects of hunger and weight gain. After 2 months she stopped talking about being overweight; according to her drawings, her body self-image changed towards normality. Now she weighs $53 \mathrm{~kg}$ and feels herself completely healthy. She still receives $5 \mathrm{mg}$ olanzapine daily.
A 30-year-old woman with anorexia nervosa since she was 18 years old additionally developed bulimia nervosa 5 years before presentation to our clinic. She was treated traditionally for many years without significant positive results. When she applied to our clinic her weight was $44 \mathrm{~kg}$, height $167 \mathrm{~cm}$. Mental state examination revealed severe body self-image disturbance. We commenced her on $5 \mathrm{mg}$ olanzapine daily. In 9 months her weight was $53 \mathrm{~kg}$, and she recovered from symptoms of both anorexia nervosa and bulimia nervosa. According to her drawings her body self-image changed from a fat little girl to an attractive grown-up woman. She still receives $5 \mathrm{mg}$ olanzapine daily.

A 34-year-old woman had been suffering from anorexia nervosa and borderline personality disorder since puberty. When she came to our clinic her weight was $60 \mathrm{~kg}$, height $180 \mathrm{~cm}$. She complained of confusion, having too many thoughts in her head at one time. She had a seriously disturbed body image - in her drawings she looked like a little fat girl without hands and secondary sexual signs. She was started on olanzapine $5 \mathrm{mg}$, and after 2 months her body image has improved (she now sees herself as a grown-up woman) and she feels healthy.

In all three cases olanzapine was well tolerated. Our patients restored their body weight and appetite as well as their body self-image. They now think of themselves as normal, mature adults. The problem is to convince the patient to start and to continue with olanzapine therapy within the first 2 months, because it takes a few weeks before a full antipsychotic effect is achieved. We think that further investigations in this area should take place.

Hansen, L. (1999) Olanzapine in the treatment of anorexia nervosa (letter). British Journal of Psychiatry, 175, 592.

Kaye, W., Strober, M., Stern, D., et al (1999) New directions in treatment research of anorexia and bulimia nervosa. Biological Psychiatry, 45, 1285-1292.

V. S. Jensen, A. Mejlhede Vesterbrogade 18, 2.th, 9400 Norresundby, Denmark

\section{Nicotine reduction: effectiveness of bupropion}

Hayford et al (1999) and others have found sustained-release bupropion useful as part of nicotine reduction treatment programmes. However, our clinical experience suggested the primary benefit of sustained-release bupropion $(300 \mathrm{mg} /$ day) occurred within the first month of treatment and the recommended second month of medication was probably not helpful.

We evaluated the treatment progress of 74 (30 one-month, 44 two-month) research volunteers (further details available from the first author upon request). Treatment condition assignment was random but one-month patients had the option of an additional two months of medication if they were not successful at quitting at immediate follow-up, thus decreasing the number of one-month participants for this study. Volunteers had a mean age of 56 and were primarily male $(91 \%)$, cigarette smokers (96\%) and White (92\%). Oneand two-month groups did not differ significantly on a number of treatment motivation measures, demographic variables and self-reported health variables.

All patients signed consent forms and received the same behavioural treatment information in the first session. All were also instructed to return to a second meeting at which time their progress was evaluated and they received more behavioural strategy information and their assigned sustained-release bupropion. Participants were followed (a) as soon as convenient after nicotine quit date, (b) three-months after immediate follow-up, and (c) six-months after initial group meeting. About $80 \%$ of potential subjects volunteered for this project and follow-up return rate averaged $81 \%$.

Use of one or two months of bupropion did not significantly affect self-reported quit rates at immediate, three-month or six-month follow-up periods. Self-reports of decreased nicotine intake among patients who did not quit entirely also did not differ between one- and two-month groups at immediate, three-month, or six-month followup periods. Only weight gain was associated with six-month treatment success. Participants who were successful reported more weight gain than those who were not. However, one- or two-month dosing schedule was not significantly associated with reported weight changes. Quitting success at six months was not related to age, gender, tobacco type, income category, race, combat exposure, years' service, sideeffect ratings, health problem ratings concerning breathing, heart, general medical, psychiatric, or substance dependence areas, religious behaviour, immediate weight gain, or initial self-ratings of treatment programme helpfulness/motivation/ self-control/completion. 
Our programme evaluation results suggest that one-month use of sustainedrelease bupropion (300 $\mathrm{mg} / \mathrm{day}$ ) is as effective as two-month use in American veterans. One-month use reduced cost but had no clear effect on general (yes/no) side-effect ratings. Future programme evaluation efforts may increase both the population size and sophistication of follow-up procedures. In addition, it may be useful to evaluate whether $15 \mathrm{mg}$ of bupropion daily may be just as effective as $300 \mathrm{mg}$ daily. Over two-month periods, previous research suggests that neither the antidepressant (Reimherr et al, 1998) nor nicotine reduction (Hurt et al, 1997) effects of bupropion were significantly different between these two dosing schedules, even with patients with a history of major depression (Hayford et al, 1999).

Hayford, K. E., Patten, C. A., Rummans, T. A., et al (1999) Efficacy of bupropion for smoking cessation in smokers with a former history of major depression or alcoholism. British Journal of Psychiatry, 174, 173-178.

Hurt, R. D., Sachs, D. P., Glover, E. D., et al (1997) A comparison of sustained-release bupropion and placebo for smoking cessation. New England Journal of Medicine, 337, $1195-1202$.

Reimherr, F. W., Cunningham, L. A., Batey, S. R. et al (1998) A multicenter evaluation of the efficacy and safety of 150 and $300 \mathrm{mg} / \mathrm{d}$ sustained-release bupropion tablets versus placebo in depressed outpatients. Clinical Therapeutics, 20, 505-516.

J. K. Neumann, B. Peeples, J. East, A. R. Ellis VA Medical Center, PO Box 4000, Mountain Home, TN 37684, USA

\section{Paradoxical pattern of haematological risk with clozapine}

I would be intrigued to hear further comments from Munro et al (1999) concerning the apparent paradox of the inverse relationship between dose and risk, both of neutropenia and of agranulocytosis.

A curious interaction of enzymes and metabolites, as briefly alluded too, is a fascinating possibility; other more banal explanations might also be entertained. One imagines the authors considered the possibility of an artefact. The agranulocytosis risk was the raison d'être for the Clozaril Patient Monitoring Service (CPMS); so we may reasonably propose a tendency to (a) reduce the dose, and (b) fail to raise it, in those who exhibited lower white cell counts. Could such a mechanism produce these results? The data presented do not appear sufficient to rule out such an explanation.
Do the authors wish to comment on why the baseline white blood cell count should be associated with hazard of neutropenia but seemingly (from absence of specific data and comment) not with agranulocytosis.

Psychiatrists have, over the years, made minimal use of therapeutic drug monitoring and one presumes from this report that this was not incorporated in any way into the CPMS. Is there a lesson here and might that have elucidated the putative 'ratio of metabolites' question?

Munro, J., O'Sullivan, D., Andrews, C., et al (1999) Active monitoring of 121760 clozapine recipients in the UK and Ireland. Beyond pharmacovigilance. British Journal of Psychiatry, 175, 576-580.

K. Gillman James Cook University, Tropical Psychopharmacology Research Unit, PO Box 8183, Mount Pleasant QLD 4740, Australia

Authors' reply: We are grateful for the above comments and for the chance to cover the issue of clozapine dose and blood dyscrasias in more detail. Extensive analyses were done to explore this and the key message is that there is no evidence that risk of haematological discontinuation is related to increased dose.

The peak risk for both agranulocytosis and neutropenia on clozapine occurs in weeks $6-18$ of treatment. It is likely that in these early stages of treatment, the dose of clozapine is still being titrated up to a typical maintenance level. The low doses of clozapine in patients with agranulocytosis or neutropenia reflect the overlap in time of peak risk for blood dyscrasias and the drug titration period. This is more likely to explain the finding, rather than the proposed tendency to reduce dose or fail to raise it in those who exhibit lower white cell counts. When it is noted that a patient's white blood cell (WBC) count is falling, a course of action (e.g. repeat blood monitoring) is advised by the CPMS. This does not include any advice about reductions in or maintenance of clozapine dose, because the blood problems associated with the drug have been repeatedly reported as being dose-independent phenomena.

Low baseline WBC count was associated with increased hazard of neutropenia, but not agranulocytosis. It is likely that patients discontinuing clozapine for neutropenia have a number of different aetiologies behind the reduced WBC count. There is a natural variance in WBC count within the population. Those individuals tending to have a low count pre-treatment were more likely to be excluded for subsequent low counts coincidental to the clozapine treatment. This is shown by the expected finding that African-Caribbean patients have lower baseline WBC counts than Caucasians because of benign ethnic variation and, correspondingly, have higher rates of neutropenia on clozapine treatment. In sharp contrast, the rate of agranulocytosis in African-Caribbean patients is not increased. Frequency of agranulocytosis is clearly independent of the baseline WBC count, suggesting that different mechanisms exist to explain the neutropenia and agranulocytosis.

Owing to the editorial requirements to shorten the original draft, the discussion around the ratio of drug metabolites had to be truncated. The excellent suggestion regarding therapeutic drug monitoring and elucidation of the metabolite ratio, as a possible key to discovering the mechanism, points to a possible lost opportunity. Therapeutic drug monitoring is not undertaken or required routinely. However, if requested, the parent drug and the major metabolites can be measured. Unfortunately, these easily measured metabolites are very unlikely to yield important information regarding toxic mechanism, although they are proving useful in assisting with a variety of specific clinical situations such as drug interaction and suspected compliance problems. The characteristics of these major metabolites, present almost invariably in every patient at generally constant ratios, simply does not explain the frequency and temporal patterns seen for agranulocytosis. Agranulocytosis is unlikely to be due to the direct toxicity of the parent drug or these major stable metabolites. A more promising possibility involves the formation of a short-lived reactive metabolite, a nitrenium ion which binds to neutrophil proteins. This may be the mediator of the toxicity by disruption of neutrophil function or by acting as a hapten to invoke immune destruction of the neutrophil. The explanation of why only $0.73 \%$ develop agranulocytosis still has to invoke multifactorial possibilities based on individual differences in bioactivation and detoxification, which may be genetically determined.

R. Kerwin, J. Munro Institute of Psychiatry, De Crespigny Park, Denmark Hill, London SE5 8AF

D. O'Sullivan Novartis Pharmaceuticals UK Ltd, Frimley, Surrey 